\title{
ISOTROPY IN THE TWO-POINT ANGULAR CORRELATION FUNCTION OF THE COSMIC MICROWAVE BACKGROUND
}

\author{
SOPHIE ZHANG \\ Department of Physics, University of Michigan 450 Church St, Ann Arbor, MI 48109-1040, USA; sophz@umich.edu \\ Received 2011 October 24; accepted 2012 February 23; published 2012 March 7
}

\begin{abstract}
We study the directional dependence of the angular two-point correlation function in maps of the cosmic microwave background (CMB). We propose two new statistics: one which measures the correlation of each point in the sky with a ring of points separated an angle $\theta$ away, and a second one that measures the missing angular correlation above $60 \mathrm{deg}$ as a function of direction. Using these statistics, we find that most of the low power in cut-sky maps measured by the Wilkinson Microwave Anisotropy Probe experiment comes from unusually low contributions from the directions of the lobes of the quadrupole and the octupole. These findings may aid a future explanation of why the CMB exhibits low power at large angular scales.
\end{abstract}

Key words: cosmic background radiation - methods: data analysis - methods: statistical

Online-only material: color figures

\section{INTRODUCTION}

With the launch of the Wilkinson Microwave Anisotropy Probe (WMAP) in 2003, the CMB has been measured in highly detailed full-sky maps (Bennett et al. 2003; Spergel et al. 2007; Hinshaw et al. 2009; Jarosik et al. 2011), which have been examined in great detail over the past few years (Cruz et al. 2005; de Oliveira-Costa et al. 2004; Land \& Magueijo 2005; Hoftuft et al. 2009; Hansen et al. 2009; Kim \& Naselsky 2010; Bennett et al. 2011). In particular, the angular two-point correlation function $C(\theta)$ has been studied; it is defined as the average product between the temperature of two points an angle $\theta$ apart

$$
C(\theta)=\left.\overline{T\left(\hat{\Omega}_{1}\right), T\left(\hat{\Omega}_{2}\right)}\right|_{\hat{\Omega}_{1} \cdot \hat{\Omega}_{2}=\cos (\theta)} .
$$

Here, $T(\hat{\Omega})$ is the fluctuation around the mean of the temperature in direction $\hat{\Omega}$ on the sky. Several anomalies have been claimed in the angular correlation function, especially the missing power on large angular scales (for a review, see Copi et al. 2010). Specifically, the angular correlation function is very nearly zero at scales above $60^{\circ}$; such a low correlation has a significance of $\geqslant 3.2 \sigma$ in the standard Gaussian random, statistically isotropic cosmology. This result, first observed in $C O B E$ data, has been strengthened with WMAP first-year data (Hinshaw et al. 1996), as well as later WMAP observations (Copi et al. 2007, 2009; Sarkar et al. 2011), though questions have been raised regarding its significance (Pontzen \& Peiris 2010; Efstathiou et al. 2010; Ma et al. 2011)

In this brief report, we explore the directional contributions to the angular two-point correlation function. Directional information is lost in the original definition of $C(\theta)$, as Equation (1) assumes statistical isotropy and averages over all directions on the sky. Our goal is to provide a generalization of Equation (1), and ascertain if there are specific directions that result in the unusually low correlation.

\section{FIXED-VERTEX $C(\theta)$}

The angular two-point correlation function is defined by Equation (1), where the average is taken over all pairs of points separated by an angle $\theta$ on the sky.
We now propose a new statistical quantity, a fixed-vertex correlation function:

$$
C(\hat{\Omega}, \theta)=\left.\overline{T(\hat{\Omega}), T\left(\hat{\Omega}_{2}\right)}\right|_{\hat{\Omega} \cdot \hat{\Omega}_{2}=\cos (\theta)},
$$

where $\hat{\Omega}$ is a direction in the sky, and the average is taken over a ring of pixels separated by $\theta$ from the direction $\hat{\Omega}$. In particular, notice that $C(\theta)=(1 / N) \sum_{i=1}^{N} C\left(\hat{\Omega}_{i}, \theta\right) .{ }^{1}$ Our new statistic preserves directional information that is normally lost when averaging over the entire $\mathrm{sky}^{2}$; as a result, it may be used to test isotropy. Maps of $C(\hat{\Omega}, \theta)$ for $\theta=60^{\circ}$ and $\theta=120^{\circ}$ are shown in Figure 1.

In order to quantify the low power in the angular two-point function between approximately $60^{\circ}$ and $180^{\circ}$, the statistic (Spergel et al. 2003)

$$
S_{1 / 2}=\int_{-1}^{1 / 2} C(\theta)^{2} d(\cos \theta)
$$

has been widely used. In order to examine possible anisotropies in $C(\theta)$, we attempt to separate $S_{1 / 2}$ into contributions from specific directions $\hat{\Omega}$; we define

$$
S_{1 / 2}(\hat{\Omega}) \equiv \frac{1}{N} \int_{-1}^{1 / 2} C(\hat{\Omega}, \theta) C(\theta) d(\cos \theta),
$$

so that the sum of $S_{1 / 2}(\hat{\Omega}, \theta)$ over all pixels is $S_{1 / 2}{ }^{3}$

\footnotetext{
1 On a masked sky, the standard $C(\theta)$ is no longer the simple average of $C(\hat{\Omega}, \theta)$. We need to introduce the weight function $W(\hat{\Omega}, \theta)$, the fraction of unmasked points in the ring $\theta \operatorname{deg}$ away from $\hat{\Omega}$. Thus,

$$
C(\theta)=\left.\frac{\sum_{i=1}^{N} C\left(\hat{\Omega}_{i}, \theta\right) W\left(\hat{\Omega}_{i}, \theta\right)}{\sum_{i=1}^{N} W\left(\hat{\Omega}_{i}, \theta\right)}\right|_{\hat{\Omega}_{1} \cdot \hat{\Omega}_{2}=\cos (\theta)} .
$$

2 As a simple example, consider if the sky were rotated by some amount. In this case, standard statistics such as $C(\theta)$ and the $C_{l}$ s would remain identical, but $C(\hat{\Omega}, \theta)$ would be similarly rotated.

3 For a masked sky, defining $W\left(\hat{\Omega}_{i}, \theta\right)$ as the fraction of the ring for the respective $C\left(\hat{\Omega}_{i}, \theta\right)$ that is unmasked (W is 0 if $\Omega$ is a masked point) we have

$$
S_{1 / 2}\left(\hat{\Omega}_{i}\right)=\int_{-1}^{1 / 2} \frac{W\left(\hat{\Omega}_{i}, \theta\right) C\left(\hat{\Omega}_{i}, \theta\right) C(\theta) d(\cos \theta)}{\sum_{j=1}^{N} W\left(\hat{\Omega}_{j}, \theta\right)} .
$$



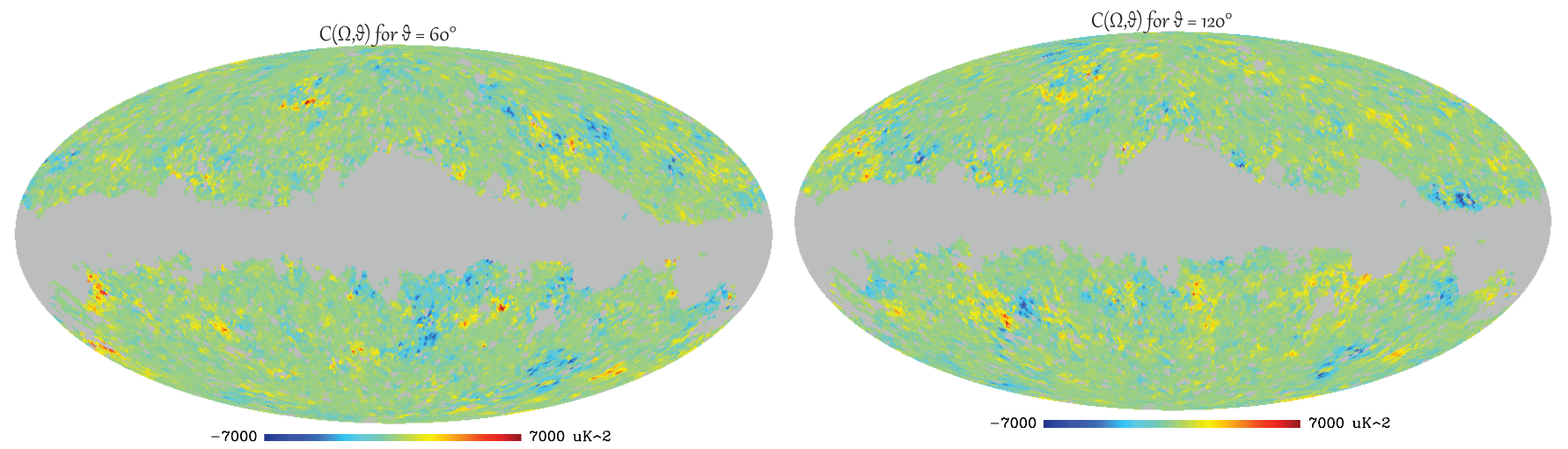

Figure 1. Full-sky maps of the fixed-vertex correlation function $C(\hat{\Omega}, \theta)$ for $\theta=60^{\circ}$ (left panel) and $\theta=120^{\circ}$ (right panel) at resolution $N_{\text {side }}=64$, calculated for the cut-sky ILC map.

(A color version of this figure is available in the online journal.)

Note that the quantity $S_{1 / 2}(\hat{\Omega})$ is non-local, in that it receives contributions from not only the direction $\hat{\Omega}$, but from the whole sky, due to the term $C(\theta)$ in Equation (5). So, for example, excluding a pixel $\hat{\Omega}$ will not change the value of $S_{1 / 2}$ simply by $-S_{1 / 2}(\hat{\Omega})$, because there is also an indirect effect of the changed global $C(\theta)$ as well as the changed monopole that is being subtracted. These two are approximately constant if only a small area of the sky is affected, so analysis of $S_{1 / 2}(\hat{\Omega})$ is suitable for examination of the effect of small regions of the sky on $S_{1 / 2}$. However, these indirect effects must be taken into consideration while examining much larger regions.

\section{DIRECTIONAL CONTRIBUTIONS TO $S_{1 / 2}$}

\subsection{Random Map Analysis}

We first examine plots of $S_{1 / 2}$ in random maps to obtain an idea of what to expect. We generate 80,000 synthetic Gaussian random statistically isotropic maps, based on the underlying best-fit $\Lambda$ CDM cosmological model (Larson et al. 2011).

A strong relationship between contributions to power in $S_{1 / 2}$ and the regions of the quadrupole and octupole (QO regions for simplicity) is consistently seen in random maps. An example may be seen in the right panels of Figures 3 and 4 with the full-sky Internal Linear Combination (ILC) map.

Motivated by this result, we seek to quantify these contributions by repeating our analysis of $S_{1 / 2}(\hat{\Omega})$ in only the regions of the sky containing the quadrupole and octupole. We compute $S_{1 / 2}(\hat{\Omega})$ in a "sky" containing only the quadrupole and octupole of each map, and define the QO region to correspond to pixels $\hat{\Omega}_{\mathrm{QO}}$ where $\left|S_{1 / 2 \mathrm{QO}}\left(\hat{\Omega}_{\mathrm{QO}}\right)\right| \geqslant x ; x$ is selected so that $\sum S_{1 / 2}\left(\hat{\Omega}_{\mathrm{QO}}\right) / \sum S_{1 / 2}(\hat{\Omega}) \approx 0.9$. We define the QO region of each individual synthetic map separately. The contribution of $S_{1 / 2}$ from the QO region is thus defined as

$$
S_{1 / 2}^{\mathrm{QO}} \equiv \sum_{i} S_{1 / 2}\left(\hat{\Omega}_{i}^{\mathrm{QO}}\right)
$$

There is an extremely strong, almost linear relationship between $S_{1 / 2}^{\mathrm{QO}}$ and total $S_{1 / 2}$, plotted in Figure 2. In contrast, the total $S_{1 / 2}$ and the contributions from non-QO regions of the sky are essentially uncorrelated, except at very high $\left(\geqslant 10^{5}(\mu \mathrm{K})^{4}\right)$ values of $S_{1 / 2}$, with which we are not concerned. Note that by definition, $S_{1 / 2} \equiv S_{1 / 2}^{\mathrm{QO}}+S_{1 / 2}^{\mathrm{non}-\mathrm{QO}}$.

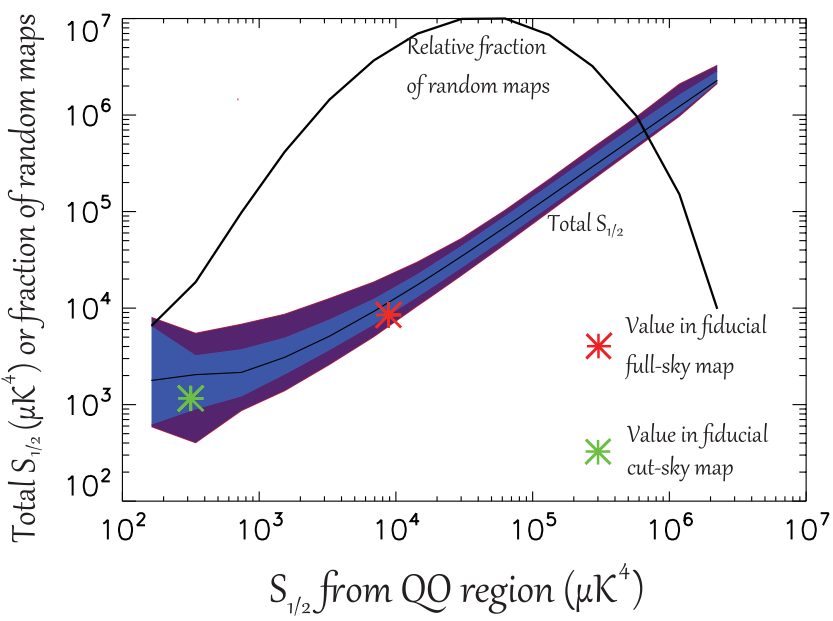

Figure 2. Relationship between $S_{1 / 2}^{\mathrm{QO}}$ and $S_{1 / 2}$ is shown. The first black curve (labeled "Total $S_{1 / 2}$ ") indicates the median relationship in random Gaussian skies. The blue and purple bands surrounding it are (respectively) 68\% and 95\% contours, indicating the spread of the distribution from cosmic variance. (Note that the error bands are only approximate for very high or low $S_{1 / 2}$ values, due to low statistics.) The narrow contours indicate a very tight relation between the two statistics. The second black curve (labeled) logarithmically plots the relative fraction of random maps, while the green and red asterisks indicate the values for $S_{1 / 2}^{\mathrm{QO}}$ and $S_{1 / 2}$ from the fiducial cut-sky and full-sky ILC map, respectively. The full-sky ILC map (red asterix) follows the relationship (shown in the right panels of Figures 3 and 4.) The cut-sky map (green asterix), which has a low value of $S_{1 / 2}$, also has a low $S_{1 / 2}^{\mathrm{QO}}$; its position in the figure is typical compared to random maps that have similarly low values of $S_{1 / 2}$. Hence, it is possible that $S_{1 / 2}$ is low because $S_{1 / 2}^{\mathrm{QO}}$ is. See the text for details.

(A color version of this figure is available in the online journal.)

This result makes intuitive sense, as the statistic $S_{1 / 2}(\hat{\Omega})$ is based off $C\left(\hat{\Omega}_{i}, \theta\right)$, which is higher when the central point $\hat{\Omega}$ is in a high-temperature region such as the QO region. More qualitatively, $S_{1 / 2}(\hat{\Omega})$ is a measure of correlation on the largest scales in the sky-which are, of course, the quadrupole and octupole.

\subsection{WMAP Map Analysis}

We now examine WMAP's seven-year maps in our analysis (Jarosik et al. 2011). We use the full sky and cut-sky ILC maps. ${ }^{4}$

\footnotetext{
4 We have checked these results for robustness by comparing to a co-added foreground-cleaned $Q-V-W$ band map, and the results there are very similar to that of the cut-sky ILC.
} 

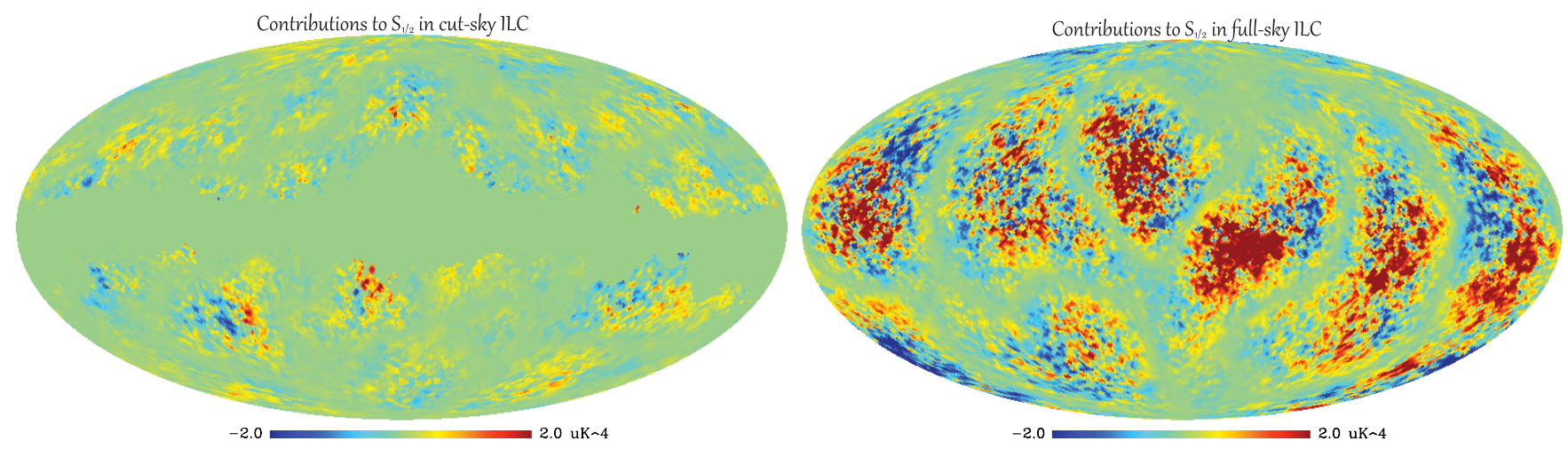

Figure 3. Quantity $S_{1 / 2}(\hat{\Omega})$ defined in Equation (5) as a function of direction $\hat{\Omega}$ in the cut-sky ILC map. The left panel shows contributions in the cut-sky map, where the masked pixels contribute nothing and are counted as zero. The right panel shows $S_{1 / 2}(\hat{\Omega})$ calculated from the full-sky ILC map. In both cases, the total value of $S_{1 / 2}(\hat{\Omega})$ summed over the entire map is mathematically equal to $S_{1 / 2}$, which is about $1000(\mu \mathrm{K})^{4}$ for the cut-sky map, and $8000(\mu \mathrm{K})^{4}$ for the full-sky map. Note the strong resemblance of the full-sky $S_{1 / 2}(\hat{\Omega})$ and the power in the quadrupole and octupole (seen in, e.g., Figure 4 in Copi et al. 2010).

(A color version of this figure is available in the online journal.)
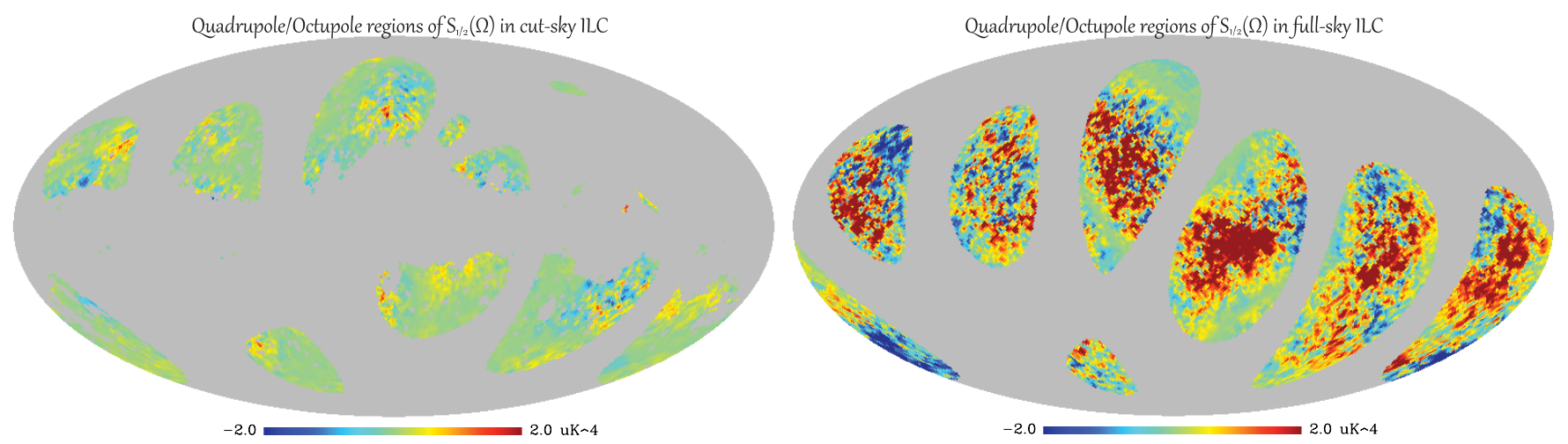

Figure 4. $S_{1 / 2}(\hat{\Omega})$ is plotted in the cut-sky (left panel) and full-sky ILC map (right panel), with only the QO regions (defined in the text) shown. Gray indicates both masked points and the non-QO region. In the full-sky map (right), correlation between the structure in $S_{1 / 2}(\hat{\Omega})$ and the QO regions is apparent, as expected. In the cut-sky map (left), there is little correlation between the structure and the QO regions, a result at odds with typical statistically isotropic random maps.

(A color version of this figure is available in the online journal.)

The maps are first degraded to $N_{\text {side }}=64$ (corresponding to pixel scale $\simeq 1^{\circ}$ ); next, the KQ75 mask is applied, and finally all resulting pixels that are more than $10 \%$ masked (i.e., with mask value of less than 0.9 ) are excluded from the analysis. Finally, the dipole was removed.

From inspection of the full-sky ILC $S_{1 / 2}(\hat{\Omega})$ (Figure 4, right panel), there is an obvious correlation between the magnitude of $S_{1 / 2}(\hat{\Omega})$ contributions and the regions where the quadrupole and octupole have power, as expected from our analysis of random maps. Quantifying this result using our previous definition of the QO region, we find that $S_{1 / 2}^{\mathrm{QO}}=8837(\mu \mathrm{K})^{4}$, while $S_{1 / 2}^{\text {non-QO }}=$ $-349(\mu \mathrm{K})^{4} .{ }^{5}$ The values observed in the full-sky map are very typical, compared to our random map results.

Moving on to the cut-sky map, there is much less power on large angular scales, as expected; only $0.06 \%$ of random maps have a similarly low value of $S_{1 / 2}$. And as the empirical relationship predicts, $S_{1 / 2}^{\mathrm{QO}}$ is similarly low, at the $0.046 \%$ level. In comparison, the non-QO contribution, $S_{1 / 2}^{\text {non-QO }}$ is quite typical

5 Note that $S_{1 / 2}^{\mathrm{QO}}$ can be greater than the full $S_{1 / 2}$, since the contributions from each individual $S_{1 / 2}\left(\hat{\Omega}_{\mathrm{QO}}\right)$ can be negative as well as positive, and their sum over the full sky is equal to the full $S_{1 / 2}$.
(15.6\% confidence level. $)^{6}$ Thus, the strong correlation that we observed in random maps continues to hold for the anomalous cut-sky map, as shown in Figure 2. What is unlikely about our sky is simply for $S_{1 / 2}^{\mathrm{QO}}$ to exhibit such a low value in the first place.

It is known that the quadrupole in the CMB sky is lower than expected from $\Lambda$ CDM results, though not anomalously so (Komatsu et al. 2011). It makes sense to consider what happens to our results above if we use the $\Lambda \mathrm{CDM}$ theoretical power spectrum for Monte Carlo simulations as before, but substituting the low WMAP quadrupole $\left(C_{2}=210(\mu \mathrm{K})^{2}\right)$, in order to test if our result is still valid. As expected, the significance of $S_{1 / 2}$ relative to these new synthetic maps is now lower $(0.70 \%$ compared to $0.06 \%$ before), and a similar effect is seen in our new statistics: $S_{1 / 2}^{\mathrm{QO}}$ has a significance of $0.58 \%$ (previously $0.046 \%$ ), and $S_{1 / 2}^{\text {non-QO }}$ is again typical-27.4\% C.L., compared to $15.6 \%$ before. Therefore, comparison to Gaussian random skies conditioned to have low $C_{2}$ gives results that are somewhat

\footnotetext{
6 Note that as we are working on large scales, the error bars for each individual statistic (from measurement errors due to pixel noise) are expected to be very small. The main source of error is cosmic variance-the dispersion of expected theoretical universes from Gaussian random theory; this is shown in the bands of Figure 2.
} 
less significant than before, which is expected since we already knew that part of the low- $S_{1 / 2}$ problem originated from low quadrupole. However, the strong correlation observed in random maps continues to hold. In addition, the various statistics are still low with $\leqslant 1 \%$ probability, even compared to skies with the low quadrupole, and the lowness is once again due to lack of power from the quadrupole and octupole regions.

\section{CONCLUSION}

In this Letter, we first introduced the fixed-vertex correlation function $C(\theta, \hat{\Omega})$, which has the potential to be a useful tool for studying the statistical isotropy of the correlation function. We then observe that the value of $S_{1 / 2}$ in random skies is correlated with an expected anisotropy in the quadrupole and octupole regions of the sky. Our sky, known to have a low $S_{1 / 2}$, also receives low contributions from its quadrupole and octupole regions. We have a bit of a chicken and egg problem here, as we cannot tell if the low $S_{1 / 2}^{\mathrm{QO}}$ caused a low $S_{1 / 2}$, or vice versa, or if both were caused by an underlying factor. It is worth considering that this result and the one of Pontzen \& Peiris (2010) may stem from the same root cause.

It is also interesting that random Monte Carlo skies (which are generated from the assumption of statistical isotropy) and the reconstructed full-sky ILC (which assumes statistical isotropy in the reconstruction process) have similar values of $S_{1 / 2}$ and $S_{1 / 2}^{\mathrm{QO}}$, while the cut-sky temperature map is anomalous. These findings may provide a hint for a future understanding of the origin of large-angle missing power in the CMB.

We thank Dragan Huterer for initial guidance in the project and many useful suggestions. We thank Glenn Starkman for introducing the idea of $C(\hat{\Omega}, \theta)$. We thank Hiranya Peiris for a useful communication. We thank an anonymous reviewer for useful suggestions and comments. We acknowledge use of the HEALPix package (Górski et al. 2005) and the Legacy Archive for Microwave Background Data Analysis (LAMBDA). This work was partly supported by NSF under contract AST-0807564, NASA under contract NNX09AC89G, and the University of Michigan.

\section{REFERENCES}

Bennett, C. L., Halpern, M., Hinshaw, G., et al. 2003, ApJS, 148, 1 Bennett, C. L., Hill, R. S., Hinshaw, G., et al. 2011, ApJS, 192, 17

Copi, C. J., Huterer, D., Schwarz, D. J., \& Starkman, G. D. 2007, Phys. Rev. D, 75, 023507

Copi, C. J., Huterer, D., Schwarz, D. J., \& Starkman, G. D. 2009, MNRAS, 399, 295

Copi, C. J., Huterer, D., Schwarz, D. J., \& Starkman, G. D. 2010, Adv. Astron, 2010, 847541

Cruz, M., Martínez-González, E., Vielva, P., \& Cayón, L. 2005, MNRAS, 356, 29

de Oliveira-Costa, A., Tegmark, M., Zaldarriaga, M., \& Hamilton, A. 2004, Phys. Rev., D69, 063516

Efstathiou, G., Ma, Y.-Z., \& Hanson, D. 2010, MNRAS, 407, 2530

Górski, K. M., Hivon, E., Banday, A. J., et al. 2005, ApJ, 622, 759

Hansen, F. K., Banday, A. J., Górski, K. M., Eriksen, H. K., \& Lilje, P. B. 2009, ApJ, 704, 1448

Hinshaw, G., Branday, A. J., Bennett, C. L., et al. 1996, ApJ, 464, L25

Hinshaw, G., Weiland, J. L., Hill, R. S., et al. 2009, ApJS, 180, 225

Hoftuft, J., Eriksen, H. K., Banday, A. J., et al. 2009, ApJ, 699, 985

Jarosik, N., Bennett, C. L., Dunkley, J., et al. 2011, ApJS, 192, 14

Kim, J., \& Naselsky, P. 2010, Phys. Rev., D, 82, 063002

Komatsu, E., Smith, K. M., Dunkley, J., et al. 2011, ApJS, 192, 18

Land, K., \& Magueijo, J. 2005, Phys. Rev. Lett., 95, 071301

Larson, D., Dunkley, J., Hinshaw, G., et al. 2011, ApJS, 192, 16

Ma, Y.-Z., Efstathiou, G., \& Challinor, A. 2011, Phys. Rev. D, 83, 083005

Pontzen, A., \& Peiris, H. V. 2010, Phys. Rev. D, 81, 103008

Sarkar, D., Huterer, D., Copi, C. J., Starkman, G. D., \& Schwarz, D. J. 2011, Astropart. Phys., 34, 591

Spergel, D. N., Bean, R., Doré, O., et al. 2007, ApJS, 170, 377

Spergel, D. N., Verde, L., Peiris, H. V., et al. 2003, ApJS, 148, 175 